\title{
M2-polarized tumor-associated macrophages promoted epithelial-mesenchymal transition in pancreatic cancer cells, partially through TLR4/IL-10 signaling pathway
}

\author{
Chao-Ying Liu, Juan-Ying Xu, Xiao-Yan Shi, Wei Huang, Ting-Yan Ruan, Ping Xie and Jun-Li Ding
}

M2-polarized tumor-associated macrophages (TAMs) are key regulators of the link between inflammation and cancer. A negative correlation between infiltration intensity of M2-polarized TAMs and prognosis of pancreatic cancer has been reported. Epithelial-mesenchymal transition (EMT) is an important biological process in the progression of primary tumors toward metastasis. Inflammation-induced EMT has been previously shown, therefore, we hypothesized M2polarized TAMs could induce EMT in pancreatic cancer. Toll-like receptor 4 (TLR4) signaling has an active role in tumor progression during chronic inflammation and the receptor is primarily expressed on macrophages. Activation of TLR4 on M2-polarized TAMs stimulates an increase in the cytokine interleukin-10 (IL-10); consequently, another aim was to investigate the potential role of TLR4/IL-10 signaling in the EMT of pancreatic cancer. Treatment with IL-4 (20 ng/ml) for $24 \mathrm{~h}$ successfully induced the polarization of macrophage cell line RAW 264.7 to M2 phenotype, IL-10 ${ }^{\text {high }}$, IL- $12^{\text {low }}$, and $\mathrm{IL}-23^{\text {low }}$, and high expression of CD204 and CD206. A coculture system allowed investigation of the roles of M2-polarized TAMs and TLR4/IL-10 signaling in the EMT of Panc- 1 and BxPC-3 pancreatic cancer cell lines. Our results showed that coculture with M2-polarized TAMs increased fibroblastic morphology, upregulated mesenchymal markers vimentin and snail at the mRNA and protein levels, and increased proliferation, migration, and metalloproteinase (MMP)2 and MMP9 proteolytic activity in pancreatic cancer cells. Simultaneously, coculture with M2-polarized TAMs decreased the expression of the epithelial marker E-cadherin. Coculture with pancreatic cancer cells increased TLR4 mRNA and protein expression in M2-polarized TAMs. Application of TLR4 siRNA and neutralizing antibodies against TLR4 and IL-10 markedly inhibited E-cadherin reduction and the upregulation of snail and vimentin. Furthermore, activation of TLR4 signaling by lipopolysaccharide profoundly increased the EMT of pancreatic cancer cells. In conclusion, M2-polarized TAMs promoted EMT in pancreatic cancer cells partially through TLR4/IL-10 signaling, suggesting novel therapeutic strategies and enhancing our understanding of M2-polarized TAMs.

Laboratory Investigation (2013) 93, 844-854; doi:10.1038/labinvest.2013.69; published online 10 June 2013

KEYWORDS: epithelial-to-mesenchymal transition; IL-10; M2-polarized TAMs; pancreatic cancer; Toll-like receptor 4

Pancreatic ductal adenocarcinoma is highly malignant and is resistant to chemo- and radiotherapeutics. ${ }^{1}$ Recent evidence suggests the inflammatory environment of the tumor is critical for its progression. ${ }^{2}$ Tumor-associated macrophages (TAMs) are derived from circulating monocytes, which are highly abundant within the tumor and provide a key link between inflammation and cancer. $^{3}$ TAMs develop a phenotype similar to M2-polarized macrophages and respond to recruitment to the tumor microenvironment. ${ }^{4}$ Previous studies have commonly associated elevated numbers of TAMs with tumor progression and poor patient outcome. ${ }^{5}$ This association is likely linked to the typical presence of M2-polarized TAMs during cancer invasion, ${ }^{6}$ however, the exact mechanisms by which these cells influence the progression of pancreatic cancer are not clear.

The transition of epithelial-to-mesenchymal cells (EMT) is highly important for morphological changes to occur during embryonic development, and accumulating evidence suggests it also contributes to the progression of primary tumors toward a metastatic state. ${ }^{7}$ Epithelial cell markers, such as

Department of Medical Oncology, Wuxi People's Hospital, Affiliated to Nanjing Medical University, Wuxi, PRC

Correspondence: Dr J-L Ding, PhD, Department of Medical Oncology, Wuxi People's Hospital, Affiliated to Nanjing Medical University, No. 299, Qingyang Road, Wuxi 214023, PRC.

E-mail: Dingjunliletters@163.com

Received 6 September 2012; revised 13 April 2013; accepted 23 April 2013 
E-cadherin, are diminished during EMT while mesenchymal cell markers are upregulated. Transcription factors that repress E-cadherin expression, including Snail and Slug, are involved in this transition, ${ }^{8}$ and EMT markers have been identified in tumor tissue following pancreatic resection. ${ }^{9}$ Macrophages within tumor tissue greatly contribute to the aggressive progression of cancer by releasing cell-stimulating growth factors and cytokines. ${ }^{3}$ Such factors, including interleukin-6 (IL-6) and tumor necrosis factor- $\alpha$ (TNF- $\alpha$ ) may activate EMT and enhance metastasis. ${ }^{10}$ Imaging data suggest EMT is involved in migration of carcinoma cells from primary tumors, and the inflammatory microenvironment promoted by cells including TAMs is necessary for this process. ${ }^{11}$ Therefore, we hypothesize that macrophages could induce EMT and promote the progression of pancreatic cancer.

Toll-like receptors (TLRs) are important in innate immunity and are primarily expressed on macrophages and dentritic cells. Cytokine secretion occurs upon TLR activation on these cells, inducing an inflammatory response. Of this class of receptors, TLR4 has been most closely connected to inflammation-mediated carcinogenesis and tumor progression. ${ }^{12}$ TLR4 signaling inhibition in TAMs was shown to reduce cytokines and weaken their tumor-promoting activity in experimental lung metastasis. ${ }^{13}$ Despite this evidence, there is still no direct correlation between TLR4 signaling and EMT. A recent report suggested activation of TLR4 signaling on M2-polarized TAMs stimulates an increased release of IL-10. ${ }^{14}$ The positive correlation between serum IL-10 levels and cancer progression and the localization of IL-10 in advanced metastases proposes IL-10 has a crucial role within the tumor. ${ }^{15,16}$ As such, one of the aims in the present study is to investigate the potential role of TLR4/IL-10 signaling in the EMT of pancreatic cancer.

\section{MATERIALS AND METHODS Antibodies and Reagents}

Mouse anti-E-cadherin, mouse anti-vimentin, mouse anti-TLR4, and goat anti-snail antibodies were purchased from Santa Cruz Biotechnology (Santa Cruz, CA). Mouse anti-CD204 antibody was from TransGenic (Kumamoto, Japan). Anti-mouse TLR4 and IL-10-neutralizing antibodies $(20 \mu \mathrm{g} / \mathrm{ml})$ were obtained from eBioscience (San Diego, CA). Recombinant mouse IL-4 was from PeproTech (Rocky Hill, NJ). Other reagents were from Sigma-Aldrich (St Louis, MO) unless stated otherwise.

\section{Cell Lines and Cell Culture}

Mouse macrophage RAW 264.7 cell line and the human pancreatic cancer cell lines, BxPC-3 and Panc-1, were used in this study. All cell lines were obtained from American Type Culture Collection (Manassas, VA). RAW 264.7 and Panc-1 were cultured in Dulbecco's modified Eagle's medium (DMEM) and BxPC-3 was maintained in RPMI 1640 supplemented with $10 \%$ heat-inactivated fetal calf serum (FBS) and penicillin/streptomycin. Cells were cultured in a standard humidified incubator at $37^{\circ} \mathrm{C}$ in a $5 \% \mathrm{CO}_{2}$ atmosphere.

\section{Induction and Evaluation of M2-Polarized TAMs}

For induction of M2-polarized TAMs, RAW 264.7 cells were cultured in complete medium with $10 \%$ FBS and $20 \mathrm{ng} / \mathrm{ml}$ IL-4 for $24 \mathrm{~h}$. For M1-polarized macrophages, $10 \mathrm{ng} / \mathrm{ml}$ LPS plus $100 \mathrm{IU} / \mathrm{ml}$ IFN- $\gamma$ (PeproTech, Rocky Hill, NJ) was used in place of IL-4. After treatment, cells were washed three times with serum-free DMEM and cultured in this medium for $48 \mathrm{~h}$. After 48 - $\mathrm{h}$ serum starvation, RAW 264.7 cells were collected and used as M2-polarized TAMs, and the medium was collected and prepared as M2-polarized TAMs-conditioned medium. The production of M2-polarized TAM markers IL- $10^{\text {high }}$, IL- $12^{\text {low }}$, and IL- $23^{\text {low }}$ in the medium and the expression of scavenger receptor CD204 and mannose receptor CD206 were measured by enzyme-linked immunosorbent assay (ELISA) kits (R\&D, Minneapolis, MN) and western blot, respectively. ELISA results were obtained by a microplate reader (Bio-Rad, M550, Hercules, CA) and were expressed in units of $\mathrm{pg} / \mathrm{ml}$. To confirm the M2 induction specificity, these markers were also assessed in M1 macrophages.

\section{Indirect Coculture of Pancreatic Cancer Cells and Macrophage}

Pancreatic cancer cells BxPC- 3 and Panc- $1\left(1 \times 10^{5}\right.$ cells/well $)$ were seeded in 24-well plates (BD Bioscience, Franklin Lakes, NJ) in RPMI1640 and DMEM medium, respectively, supplemented with 10\% FBS and penicillin/streptomycin. M2-polarized TAMs $\left(3 \times 10^{5}\right.$ cells/insert $)$ were seeded into the upper chamber of a transwell of $8.0 \mu \mathrm{m}$ pore size (Corning Incorporated, Kennebunk, ME) in DMEM with $10 \% \mathrm{FBS}$ and penicillin/streptomycin. The following day, the culture insets with M2-polarized TAMs were placed into the wells of the 24-well plates containing pancreatic cancer cells, and incubated up to 3 days in RPMI1640 or DMEM medium supplemented with 1\% FBS and penicillin/streptomycin. Cells were cultured in a standard humidified incubator at $37^{\circ} \mathrm{C}$ in a $5 \% \mathrm{CO}_{2}$ atmosphere.

\section{Quantitative Real-Time Reverse Transcription PCR}

Total RNA was extracted with TRIzol reagent according to the manufacturer's instructions. Reverse transcription was performed in $20 \mu \mathrm{l}$ using $5 \mu \mathrm{g}$ total RNA and random hexamers (TaKaRa Biotechnology, Ltd, Dalian, China). One microliter of cDNA was amplified using a SYBR green I reaction system. Specific primers were designed and synthesized by TaKaRa. Real-time PCR was performed on an iCycler iQ (Bio-Rad, Hercules, CA) beginning at $95^{\circ} \mathrm{C}$ for $20 \mathrm{~s}$, followed by 40 cycles: at $95^{\circ} \mathrm{C}$ for $1 \mathrm{~s}$, and at $60^{\circ} \mathrm{C}$ for $20 \mathrm{~s}$. A dd $\mathrm{H}_{2} \mathrm{O}$ control was included for each template. All reactions were performed in triplicate in each single run. The amount of target gene mRNA of all samples was normalized to $\beta$-actin. Relative quantitative gene expression was calcu- 
Table 1 Specific primers for the E-cadherin, vimentin, snail, and $\beta$-actin genes

\begin{tabular}{|c|c|c|}
\hline Gene & Primer & Length (bp) \\
\hline \multicolumn{3}{|l|}{ E-cadherin } \\
\hline Forward & 5'-GAGTGCCAACTGGACCATTCAGTA-3' & 86 \\
\hline Reverse & 5'-CACACCAGCAGCATCACA-3' & \\
\hline \multicolumn{3}{|l|}{ Vimentin } \\
\hline Forward & 5'-GGTGGACCAGCTAACCAACGA-3' & 183 \\
\hline Reverse & 5'-TCAAGGTCAAGACGTGCCAGA-3' & \\
\hline \multicolumn{3}{|l|}{ Snail } \\
\hline Forward & 5'-GCTCCTTCGTCCTTCTCCTCTA-3' & 390 \\
\hline Reverse & 5'-GGCACTGGTACTTCTTGACA-3' & \\
\hline \multicolumn{3}{|l|}{$T L R 4$} \\
\hline Forward & 5'-ATCTCAGCAAAATCCCTCAT-3' & \\
\hline Reverse & 5'-AATCCAGCCACTGAAGTTGT-3' & 123 \\
\hline \multicolumn{3}{|l|}{$\beta$-Actin } \\
\hline Forward & 5'-TGGCACCCAGCACAATGAA-3' & 186 \\
\hline Reverse & 5'-CTAAGTCATAGTCCGCCTAGAAGCA-3' & \\
\hline
\end{tabular}

lated as previously described ${ }^{17}$ and expressed as a percentage of the monocultured cells. The sequences of PCR primers are listed in Table 1.

\section{Western Blotting}

For protein analysis, pancreatic cancer cells and M2-polarized TAMs were homogenized with lysis buffer $(50 \mathrm{mM}$ Tris $\mathrm{pH}$ 7.4, $150 \mathrm{mM} \mathrm{NaCl}, 1 \mathrm{mM}$ EDTA, $1 \%$ Triton-X 100, $1 \%$ sodium deoxycholate, $1 \mathrm{mM}$ PMSF), and centrifuged at $15000 \mathrm{~g}$ for $10 \mathrm{~min}$ at $4{ }^{\circ} \mathrm{C}$. The supernatant was collected for subsequent protein assays. Protein concentration was determined with a BCA kit (Pierce Biotechnology, Rockford, IL). The supernatant was treated with $2 \times$ SDSpolyacrylamide loading buffer at $95^{\circ} \mathrm{C}$ for $10 \mathrm{~min}$, and was separated on $10 \%$ Tris- $\mathrm{HCl}$ polyacrylamide gels by electrophoresis. Proteins were transferred to $0.45-\mu \mathrm{m}$ polyvinylidene difluoride membranes (Millipore, Billerica, MA). Afterward, membranes were blocked with $5 \%$ non-fat dry milk in TBS- $0.05 \%$ Tween 20 (TBST) for $1 \mathrm{~h}$ at room temperature, washed three times for 10 min each in TBST, and incubated with primary antibodies overnight at $4{ }^{\circ} \mathrm{C}$. All antibodies were diluted at 1:150 except for TLR4, which was diluted to1:100 in TBST plus 5\% non-fat dry milk. After three times 10-min TBST washes, membranes were incubated with peroxidase-conjugated goat anti-mouse $\operatorname{IgG}$ at 1:5000
(Bio-Rad) and rat anti-goat IgG at 1:5000 (Bio-Rad) for $1 \mathrm{~h}$ at room temperature. After washing, membranes were analyzed by enhanced chemiluminescence (Pierce Biotechnology).

\section{MTT Assay}

Cell proliferation was measured by the 3-(4, 5-dimethylthiazol-2-yl)-2, 5-diphenyltetrazolium bromide (MTT) (Sigma-Aldrich) assay. BxPC-3 and Panc-1 pancreatic cancer cell lines were seeded in 96-well plates (BD Bioscience) at a density of $5 \times 10^{3}$ cells/well in medium and supplemented with conditioned medium from M2-polarized TAMs and incubated for $24 \mathrm{~h}$. The next day, $20 \mu \mathrm{l}$ of $5 \mathrm{mg} / \mathrm{ml}$ of MTT solution was added to each well and the plates were incubated for $4 \mathrm{~h}$ at $37^{\circ} \mathrm{C}$. Next, $150 \mu \mathrm{l} 100 \%$ DMSO per well was added to solubilize the precipitate, and the plates were shaken for $10 \mathrm{~min}$. Absorbance of each well was measured on a microplate reader (Anthos ht III, Anthos Labtec Instruments $\mathrm{GmbH}$, Wals, Austria) at a wavelength of $490 \mathrm{~nm}$.

\section{Migration Assays}

Cell migration was examined by the two-chamber assay using a transwell coculture system (Corning). Briefly, M2-polarized TAMs were seeded $\left(10^{5}\right.$ cells/well) in 24 -well plates in DMEM supplemented with $10 \%$ FBS. Pancreatic cancer cells $\left(10^{4}\right.$ cells/insert), were seeded into the upper chamber of a transwell and placed on the 24-well plate containing RAW 264.7 cells in DMEM medium with 1\% FBS. After 24-h incubation with $5 \mu \mathrm{g} / \mathrm{ml}$ mitomycin $\mathrm{C}$ to inhibit proliferation, the cell suspension in the upper chamber was aspirated, and the upper surface of the filter was carefully cleaned with cotton plugs. Cells that migrated through the polycarbonate membrane were stained with crystal violet and images from five representative fields of each membrane were taken. The migratory and invasive cells within the lower chamber were counted.

\section{Substrate Gel Electrophoresis (Zymography)}

Zymography was used to detect the proteolytic activities of gelatinases matrix metalloproteinase (MMP) 2 and MMP9 from pancreatic cancer cells. Pancreatic cancer cells were homogenized with RIPA buffer, and $20 \mu \mathrm{g}$ total protein was subjected to electrophoresis under non-reducing conditions in $8 \%$ SDS-polyacrylamide gels copolymerized with $1 \mathrm{mg} / \mathrm{ml}$ gelatin. Gels were washed two times for 30 min each in $2.5 \%$ Triton-X 100 to remove residual SDS, and incubated in substrate buffer $(50 \mathrm{mM}$ Tris- $\mathrm{HCl}, 5 \mathrm{mM} \mathrm{CaCl} 2,1 \mu \mathrm{M}$ ZnCL2, $0.01 \% \mathrm{NaN} 3$, pH 7.5) overnight at $37^{\circ} \mathrm{C}$. Next the gels were stained in $0.5 \%$ Coomassie Blue $\mathrm{G}$ in $40 \%$ methanol/10\% acetic acid for $30 \mathrm{~min}$ at room temperature and destained in $40 \%$ ethanol $/ 1 \%$ acetic acid. Clear proteolytic zones indicated the presence of gelatinases at their respective molecular weights. 


\section{TLR4 Knockdown by siRNA Transfection}

M2-polarized TAMs were transfected with TLR4-specific siRNA (Santa Cruz), nonspecific scrambled (sc) RNA (Santa Cruz), or control buffer using siRNA transfection reagent (Santa Cruz) according to the manufacturer's protocol. In brief, TAMs were seeded in six-well plates in DMEM with 10\% FBS (without antibiotics) 1 day before transfection. At the time of siRNA transfection, the cells were $80 \%$ confluent. Forty-eight hours after transfection, cells were collected and processed for analysis.

\section{Statistical Analysis}

All data were expressed as mean \pm s.d. Experiments were performed at least three times with similar results. Differences between groups were evaluated by analysis of variance (ANOVA), followed by the Tukey-Kramer post hoc test. A $P$-value $<0.05$ was considered statistically significant. Student's $t$-test for two groups or one-way ANOVA and post hoc multiple comparisons (LSD test) for three or four groups were performed to evaluate the statistical significance using the SPSS 11.5 statistical software package (SPSS, Chicago, IL). Tests were two-sided, and $P<0.05$ was considered as statistically significant.

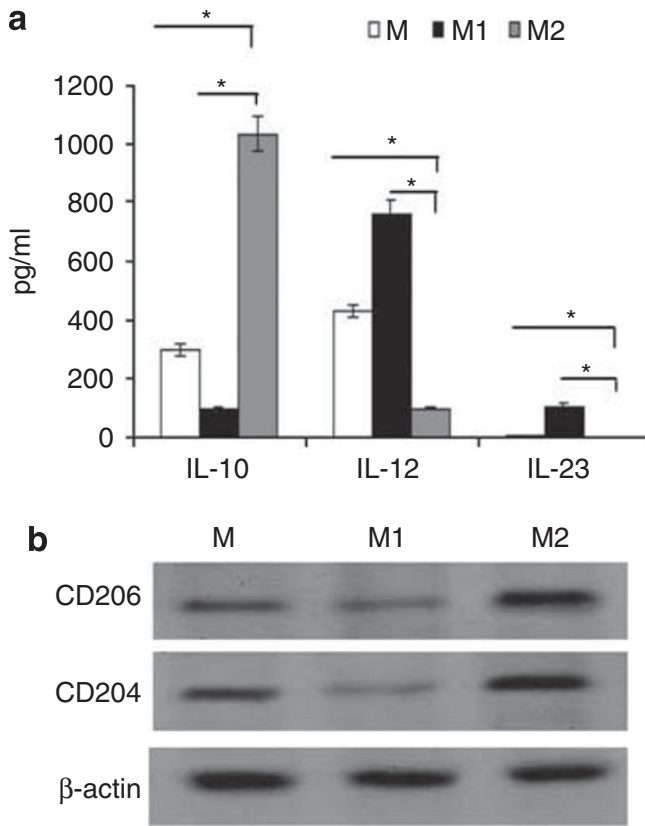

Figure 1 M2 markers were detected in native, M1, and M2 macrophages. Protein expression of CD204 and CD206 increased after treatment with interleukin-4 (IL-4). Mouse macrophage cell line, RAW 264.7, cells were treated with IL-4 $(20 \mathrm{ng} / \mathrm{ml})$ for $\mathrm{M} 2$ or $10 \mathrm{ng} / \mathrm{ml}$ lipopolysaccharide (LPS) plus $100 \mathrm{IU} / \mathrm{ml} \mathrm{IFN}-\gamma$ for $\mathrm{M} 1$ for $24 \mathrm{~h}$, then cultured in serum-free Dulbecco's modified Eagle's medium (DMEM) for $48 \mathrm{~h}$. After $48 \mathrm{~h}$, the medium was collected for enzyme-linked immunosorbent assay (ELISA) (a), and the cells were collected for western blotting (b). The data represent at least three independent experiments. ${ }^{*} P<0.05$ versus native or M1 macrophages.

\section{RESULTS}

Successful Induction of M2-Polarized TAMs

As shown in the Figure 1a, compared with native and M1 macrophages, M2-polarized TAMs secreted increased IL-10, but reduced IL-12 and IL-23. Western blotting results (Figure 1b) showed that the scavenger receptor CD204 and mannose receptor CD206 were significantly higher than native and M1 macrophages. Furthermore, M1 macrophage showed considerably reduced receptors expression than native macrophages. The results suggested that IL-4 (20 ng/ $\mathrm{ml}$ ) successfully induced the alteration of classical macrophages to M2-polarized TAMs. Next, M2-polarized TAMs were used in a coculture system.

\section{Pancreatic Cancer Cells Cocultured with M2-Polarized TAMs Exhibited Fibroblast Morphology}

Pancreatic cancer cells were monocultured or indirectly cocultured with M2-polarized TAMs, and the morphological alterations were examined under phase-contrast microscopy. Although monocultured Panc-1 cells grew in monolayer cultures, similar to epithelial clusters, Panc-1 cells cocultured with M2-polarized TAMs showed spindle fibroblastic morphology (Figures 2a and b). BxPC-3 cells cocultured with M2-polarized TAMs also underwent morphological changes similar to Panc-1 cells (Figures $2 \mathrm{c}$ and $\mathrm{d}$ ), which is characterized by morphological changes from a pebble-like shape to an elongated shape.

\section{Coculture with M2-Polarized TAMs Increased the Expression of Mesenchymal Markers but Decreased Epithelial Markers in Pancreatic Cancer Cells}

We next examined whether coculture with M2-polarized TAMs induced alterations of EMT markers for pancreatic cancer cells. Real-time PCR and western blotting showed that the mRNA and protein expressions of mesenchymal markers vimentin and snail were higher in Panc- 1 cells cocultured with M2-polarized TAMs than those in monoculture (Figures 3a-d). Conversely, the mRNA and protein expressions of epithelial marker E-cadherin were decreased in cocultured compared with monocultured Panc-1 cells (Figures $3 e$ and $\mathrm{f}$ ). Similarly, altered expression of EMT markers was also observed in BxPC-3 cells. Thus, coculture with M2-polarized TAMs increased the expression of mesenchymal markers but diminished those of epithelial markers in pancreatic cancer cells.

\section{Coculture with M2-Polarized TAMs Increased the} Proliferation and Migration of Pancreatic Cancer Cells Along with EMT, the tumor cells often exhibited accelerated proliferation and greater mobility. We next examined the effects of M2-polarized TAMs on pancreatic cancer cell proliferation and migration by MTT and two-chamber assays, respectively. MTT results showed that coculture with M2-polarized TAMs significantly increased the OD values in both Panc- 1 and BxPC-3 cells (Figure 4). As the present study 

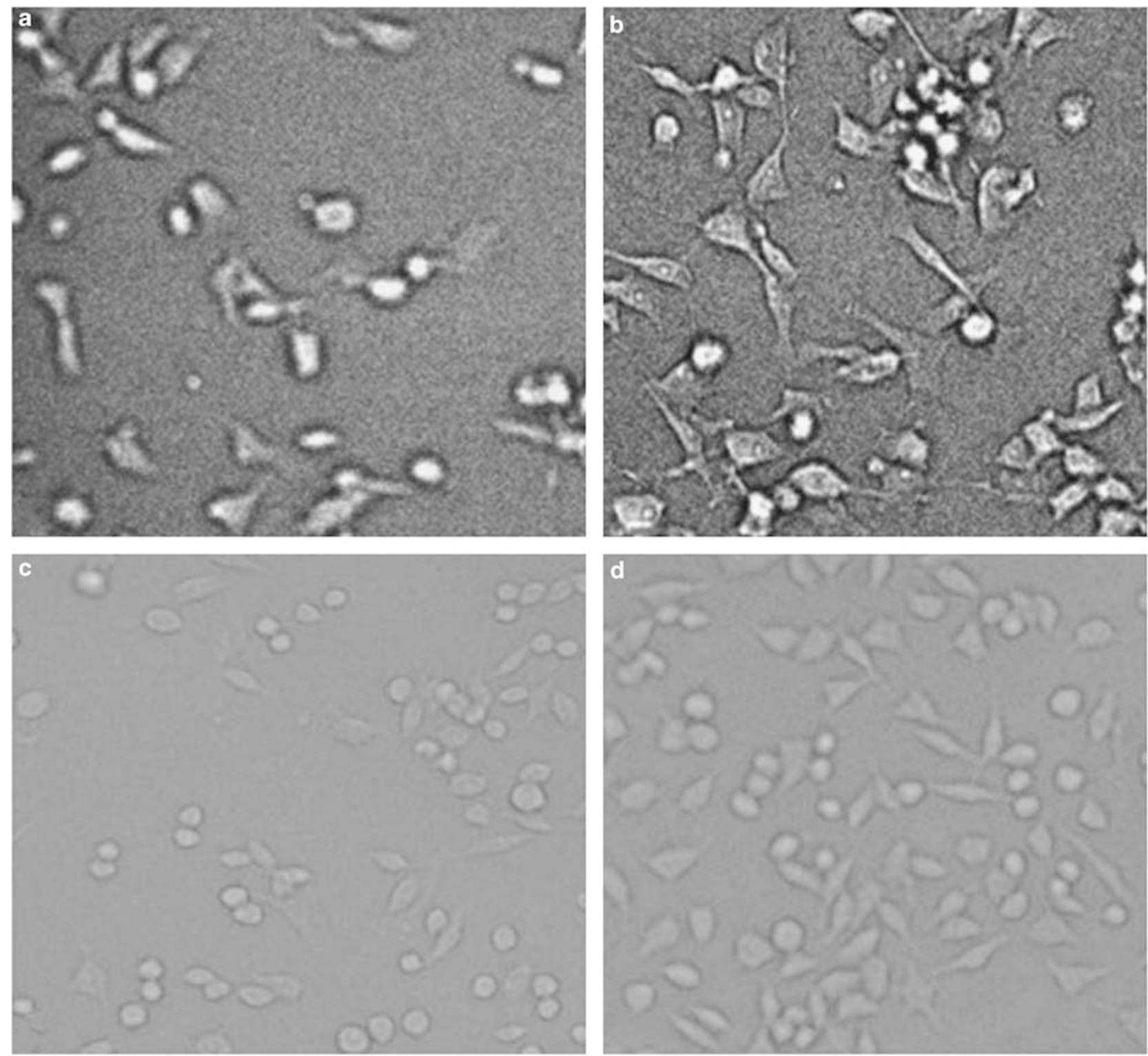

Figure 2 Coculture with M2-polarized tumor-associated macrophages (TAMs) increased the fibroblastic morphology of pancreatic cancer cells. Pancreatic cancer cell lines Panc-1 and BxPC-3 were indirectly cocultured with M2-polarized TAMs using a transwell system. After $24 \mathrm{~h}$, the cancer cells were analyzed by microscopy (original magnification, $\times 200$ ). (a) Panc-1 cells monoculture. (b) Panc-1 cells coculture. (c) BxPC-3 monoculture. (d) BxPC-3 coculture. The data represent at least three independent experiments.

demonstrated that M2-polarized TAM-induced proliferation of pancreatic cancer cells, we examined the effects of M2polarized TAMs on cancer cell migration in the presence of an inhibitor of proliferation, mitomycin C. The two-chamber migration assays showed that M2-polarized TAMs significantly promoted more Panc-1 cell migration to the lower chamber (Figures 5a-e). BxPC-3 cells cocultured with M2polarized TAMs underwent similar alterations as Panc-1 cells.

\section{Coculture with M2-Polarized TAMs Increased Proteolytic Activities of Pancreatic Cancer Cells}

MMP2 and MMP9 are known promoters of EMT via basement membrane disruption. As such, we examined the activities of MMP2 and MMP9 and the effects of M2-polarized TAMs on pancreatic cancer cell proteolytic activities by zymography. The results showed that coculture with M2-polarized TAMs significantly increased the activities of MMP2 and MMP9 in Panc-1 cells, and similar results were obtained in BxPC-3 cells (Figure 6).

\section{TLR4 Signaling has an Essential Role in EMT of Pancreatic Cancer Cells Induced by M2-Polarized TAMs}

Previous study has implicated that TLR4 on macrophages induced pancreatic cancer progression and metastasis, ${ }^{18,19}$ but it is still unknown whether TLR4 has a role in EMT. In the present study, we examined the role of TLR4 in EMT. The 

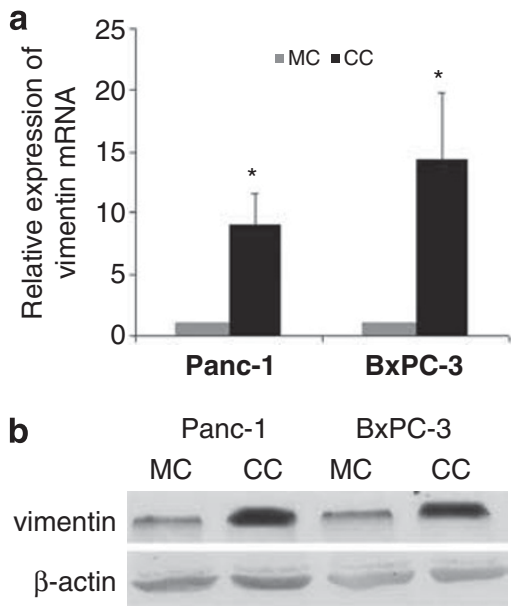

C

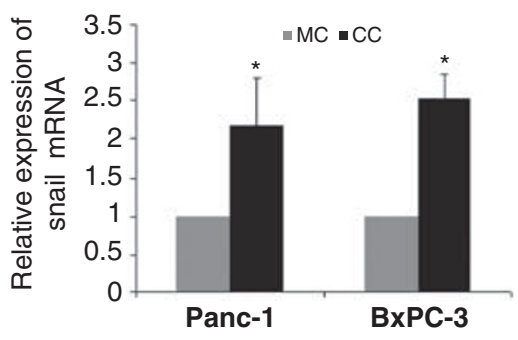

d

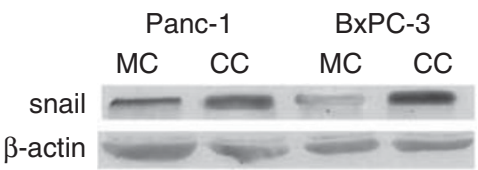

e

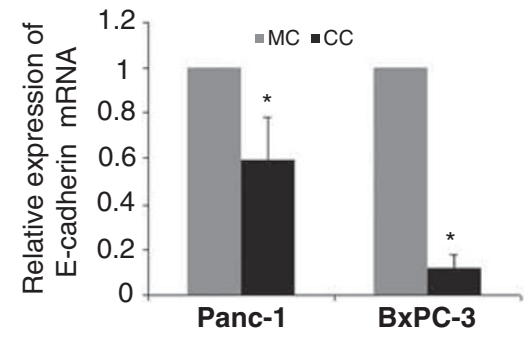

$\mathbf{f}$

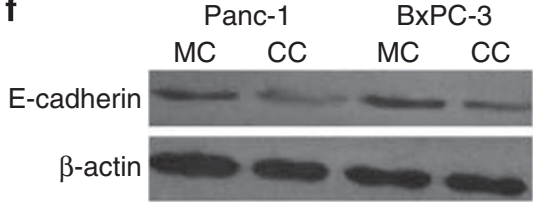

Figure 3 Coculture (CC) with M2-polarized tumor-associated macrophages (TAMs) increased the expressions of mesenchymal markers but decreased epithelial markers in pancreatic cancer cells. Panc-1 and BxPC-3 cancer cell lines were indirectly cocultured with M2-polarized TAMs using a transwell system. After $24 \mathrm{~h}$, the cancer cells were collected and analyzed. (a) Vimentin mRNA and (b) protein expression, as well as (c) Snail mRNA and (d) protein expression increased in the cell lines in CC. (e) E-cadherin mRNA and (f) protein expression decreased in the cell lines in coculture. The data represent at least three independent experiments. MC, monoculture. The data represent at least three independent experiments. ${ }^{*} P<0.05$ versus $M C$.

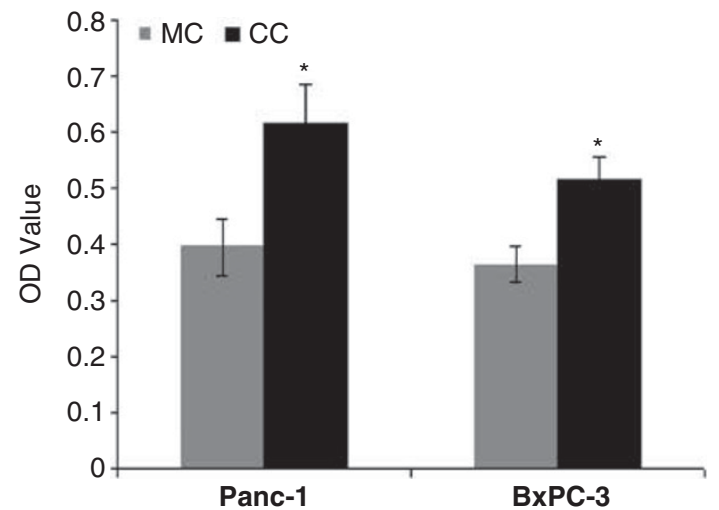

Figure 4 Coculture (CC) with M2-polarized tumor-associated macrophages (TAMs) increased the growth of pancreatic cancer cells. Pancreatic cancer cell lines Panc- 1 and BxPC-3 were cocultured with M2polarized TAMs-conditioned medium. After $24 \mathrm{~h}$, growth was assessed using an 3-(4, 5-dimethylthiazol-2-yl)-2, 5-diphenyltetrazolium bromide (MTT) assay. MC, monoculture. The data represent at least three independent experiments. ${ }^{*} P<0.05$ versus MC.

results showed that TLR4 significantly increased in both mRNA and protein levels when cocultured with pancreatic cancer cells (Figures $7 \mathrm{a}$ and $\mathrm{b}$ ), suggesting the activity of TLR4 signaling increased in M2-polarized TAMs. To examine whether TLR4 signaling contributes to the enhanced EMT induced by M2-polarized TAMs, we silenced TLR4 in these cells by RNA interference. Transfection of siRNA targeting TLR4 resulted in pronounced knockdown of mRNA (71\% in Panc-1cells and 69\% in BxPC-3 cells) and protein levels $(51.2 \%$ in Panc-1cells and $44.5 \%$ in BxPC-3 cells)
(Figures $7 \mathrm{c}$ and d). Knockdown of TLR4 resulted in significant inhibition of the reduction of E-cadherin and the increase of vimentin and snail in both Panc- 1 and BxPC-3 cells (Figures 7e and f). To confirm the role of TLR4 signaling in EMT, M2-polarized TAMs were incubated with TLR4neutralizing antibody $(10 \mu \mathrm{g} / \mathrm{ml})$ for $24 \mathrm{~h}$ before coculture with pancreatic cancer cells, after which EMT markers were quantified using western blot. Similar results were obtained with siRNA investigation. Furthermore, lipopolysaccharide (LPS), the ligand of TLR4, was also used to determine the activity of TLR4 signaling in EMT. The results showed that addition of LPS $(20 \mathrm{ng} / \mathrm{ml})$ markedly decreased E-cadherin expression, but increased the expression of vimentin and snail (Figures $7 e$ and f). These results clearly showed that M2-polarized TAMs enhanced EMT of pancreatic cancer cells partially through the TLR4 signaling pathway.

\section{IL-10-Neutralizing Antibody Inhibits M2-Polarized TAM- Induced EMT of Pancreatic Cancer Cells}

Activation of TLR4 signaling pathway is known to cause the release of various cytokines. Previous studies have shown that resting TAMs displayed higher expression of IL-10, and activation of TLR4 signaling in TAMs resulted in strong upregulation of IL-10. For the investigation of the effect of IL-10 on EMT, IL-10-neutralizing antibody $(100 \mathrm{ng} / \mathrm{ml})$ was administered. The results showed that treatment of IL-10-neutralizing antibody markedly reversed the reduction of E-cadherin and the increase of snail and vimentin (Figures $7 e$ and f). These findings clearly showed that IL-10 was the primary molecular target responsible for TLR4 function during EMT. 

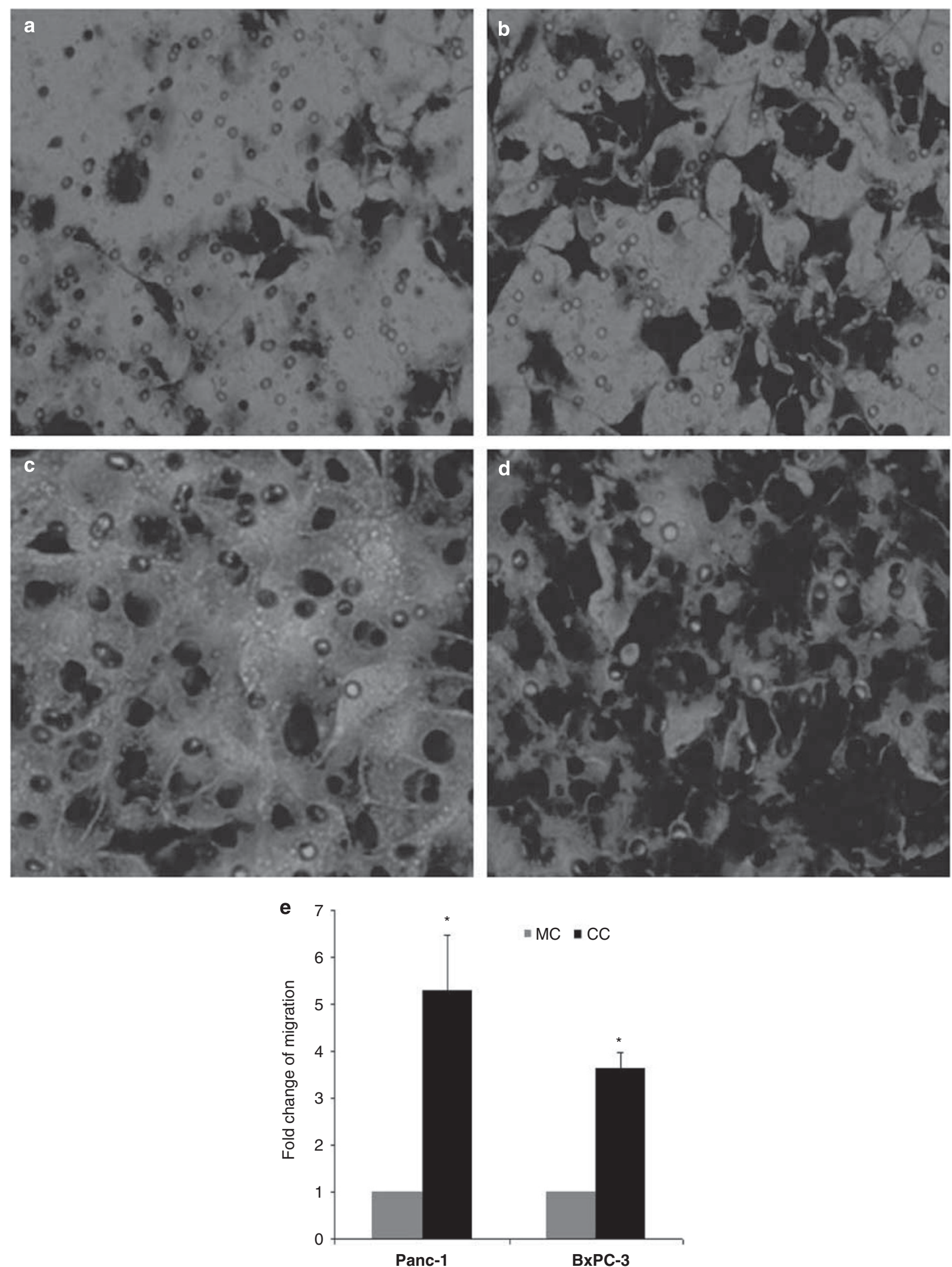

Figure 5 Coculture (CC) with M2-polarized tumor-associated macrophages (TAMs) increased the migration of pancreatic cancer cells. Panc-1 and BxPC-3 cells were indirectly cocultured with M2-polarized TAMs. After $24 \mathrm{~h}$, the cancer cells were crystal violet stained and analyzed by microscopy (original magnification, $\times 200$ ). The amount of migratory cells in the lower chamber was counted. (a) Panc-1 cell monoculture (MN). (b) Panc-1 cell CC. (c) BxPC-3 MN. (d) BxPC-3 CC. (e) The fold change of migration. The data represent at least three independent experiments. ${ }^{*} P<0.05$ versus MC. 


\section{M2-Polarized TAMs Increased Proliferation, Migration, and Proteolytic Activities of Pancreatic Cancer Cells Partially through TLR4/IL-10 Signaling}

EMT permits mobility and metastasis of cancer cells, consequently, we assessed the role of TLR4/IL-10 signaling in the proliferation, migration, and proteolytic activities of pancreatic cancer cells cocultured with M2-polarized TAMs. As shown in Figure 8a, TLR4 siRNA and neutralizing antibodies of TLR4 and IL-10 profoundly inhibited proliferation induced by M2-polarized TAMs. At the same time, LPS enhanced the proliferation. In addition, migration experiments were also performed. As shown in Figure 8b, when Panc-1 cells were cocultured with M2-polarized TAMs, the number of migrating cells increased 5.3 times and the addition of LPS elevated the migrating cell count $\sim 12$ fold compared with monocultured Panc-1 cells. On the contrary, TLR4 siRNA and neutralizing antibodies of TLR4 and IL-10 reversed the effects induced by M2-polarized TAMs. Similar results were also observed in BxPC-3 cells. Finally, proteolytic activities in cancer cells were evaluated (Figures $8 \mathrm{c}$ and d). In both Panc-1

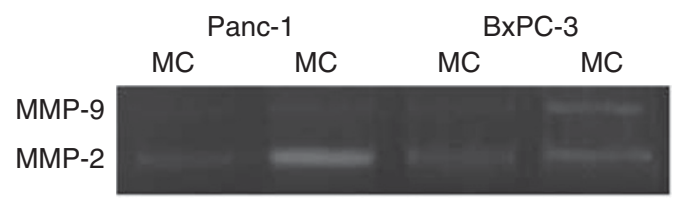

Figure 6 M2-polarized tumor-associated macrophages (TAMs) increased proteolytic activities of pancreatic cancer cells. Panc- 1 and BxPC-3 were indirectly cocultured with M2-polarized TAMs. After $24 \mathrm{~h}, \mathrm{MMP} 2$ and MMP9 proteolytic activities from pancreatic cancer cells were detected by zymography. CC, coculture; MC, monoculture. The data represent at least three independent experiments. and BxPC-3 cells, treatment with TLR4 siRNA and neutralizing antibodies of TLR4 and IL-10 significantly decreased MMP2 and MMP9 activities, conversely, LPS greatly increased the activities of both MMP2 and MMP9. These results clearly demonstrated that M2-polarized TAMs enhanced neoplastic characteristics of pancreatic cancer cells, at least in part through the TLR4/IL-10 signaling pathway.

\section{DISCUSSION}

Inflammation is a critical component of the malignant microenvironment, M2-polarized TAMs represent macrophage infiltration during chronic inflammation. TAMs and their released cytokines (eg, IL-1, TNF- $\alpha$, IL-10) may participate in all aspects of tumor cell invasion and metastasis. EMT is an important biological process of tumor progression. Recent findings suggest that TNF- $\alpha$ induces snail promoter activity and EMT in MCF-7 breast cancer cells, ${ }^{20}$ reinforcing the association between inflammation and EMT.

In this study, pancreatic cancer cell lines (Panc-1 and BxPC-3) underwent EMT phenotypic changes following coculture with M2-polarized TAMs. These results were consistent with downregulated epithelial marker expression and increased expression of mesenchymal markers. For further cellular characterization, we assessed the proliferative, migratory, and proteolytic abilities of cocultured pancreatic cancer cells compared with monocultured parental cells. Our data showed increased proliferation, migration, and proteolytic activity of pancreatic cancer cells when cocultured with M2-polarized TAMs compared with monocultured parental cells. Interestingly, we also observed that cocultured M2-polarized TAMs highly expressed TLR4 compared with monocultured M2-polarized TAMs. Furthermore, siRNA knockdown of TLR4 or inhibition of TLR4/IL-10 signaling
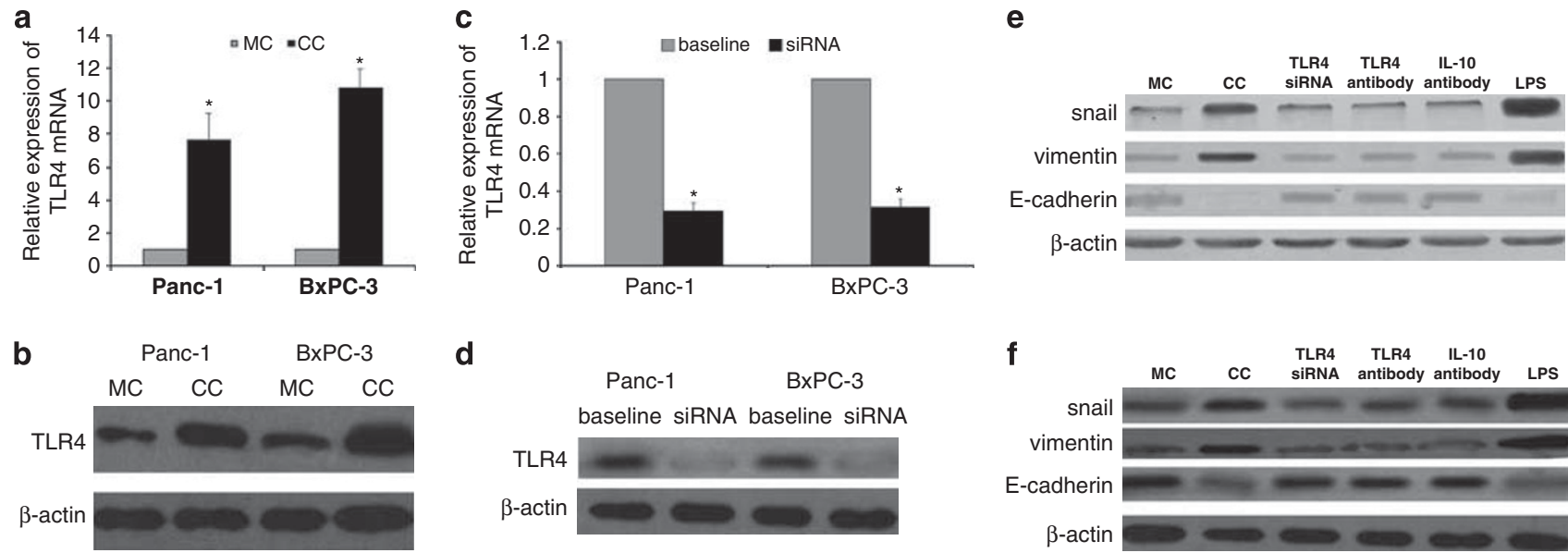

d
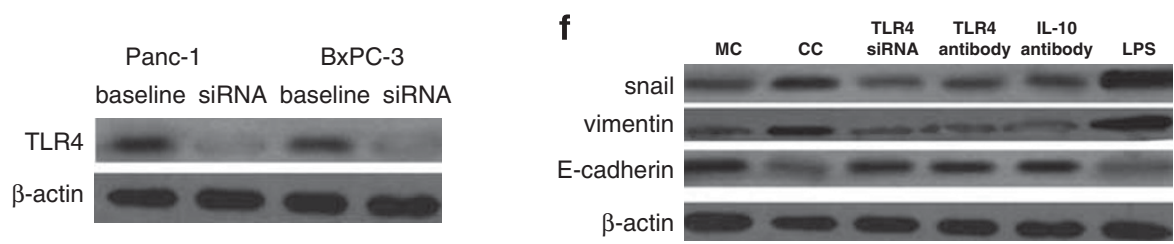

Figure 7 TLR4/interleukin-10 (IL-10) signaling has an essential role in EMT of pancreatic cancer cells induced by M2-polarized tumor-associated macrophages (TAMs). (a, b) TLR4 mRNA and protein expression in M2-polarized TAMs increased after 24-h coculture (CC) with pancreatic cancer cells. (c, d) TLR4 mRNA and protein in M2-polarized TAMs significantly decreased after transfection of TLR4 siRNA. (e, f) Knockdown of TLR4, inhibition of TLR4/IL-10 resulted in significant inhibition E-cadherin downregulation. Conversely, activation of M2-polarized TAMs by lipopolysaccharide (LPS) induced a more profound reduction of E-cadherin, but increased vimentin and snail. MC, monoculture. The data represent at least three independent experiments. ${ }^{*} P<0.05$ versus $\mathrm{MC}$ in panel $\mathbf{a},{ }^{*} P<0.05$ versus baseline in panel $\mathbf{c}$. 

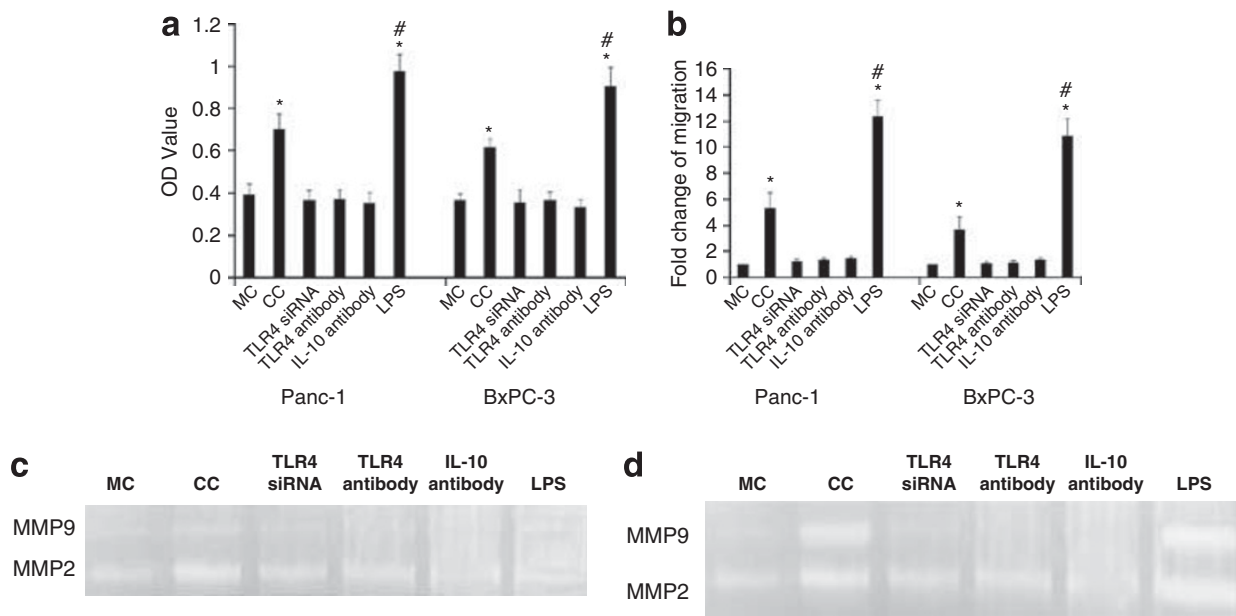

Figure 8 TLR4/interleukin-10 (IL-10) signaling has an essential role in neoplastic characteristics of pancreatic cancer cells. In coculture (CC) with M2-polarized tumor-associated macrophages (TAMs), the pancreatic cell lines were treated with either TLR4 siRNA, neutralizing antibodies of TLR4 and IL-10, and lipopolysaccharide (LPS), respectively. After $24 \mathrm{~h}$, proliferation, migration, and proteolytic activities were determined by 3-(4, 5-dimethylthiazol-2-yl)-2, 5-diphenyltetrazolium bromide (MTT) (a), transwell (b), and zymography (c, d), respectively. MC, monoculture. The data represent at least three independent experiments. ${ }^{*} P<0.05$ versus $M C,{ }^{\#} P<0.05$ versus CC.

with neutralizing antibodies markedly reversed the EMT of pancreatic cancer cells. These findings suggested that M2polarized TAMs could promote EMT and aggressive behavior in pancreatic cancer cells. Furthermore, it is possible that the M2-polarized TAM/pancreatic cancer cell interaction may dramatically activate TLR4/IL-10 signaling.

Glucocorticoids, IL-4, IL-13, IL-10, and cancer cell conditional medium are known to induce M2-polarized TAMs. ${ }^{21}$ Here, we induced macrophage cell line RAW 264.7 to M2polarized TAMs with IL-4 $(20 \mathrm{ng} / \mathrm{ml})$. In addition, treatment with IL-4 for $24 \mathrm{~h}$ induced the expression of M2 molecular markers: IL-10 ${ }^{\text {high }}$, IL- $12^{\text {low }}$, and IL-23 ${ }^{\text {low }}$, and high protein expression of CD204 and CD206.

Pancreatic cancer cell lines, including BxPC-3, Capan-1, Aspc-1, and Panc-1, are characterized as less aggressive (ie, BxPC-3 and Capan-1) or highly aggressive (ie, Aspc-1 and Panc-1) with varied degrees of EMT markers. ${ }^{22}$ For this study, we chose Panc-1 and BxPC-3. Upregulation of snail and vimentin mRNA and protein expression, and downregulation of E-cadherin occurred in two different pancreatic cancer cell lines, Panc-1 and BxPC-3, compared with monocultured parental cells. EMT has been implicated in the progression of pancreatic cancer, however, no studies have addressed whether M2-polarized TAMs induce EMT in pancreatic cancer cells. Here, we showed for the first time that indirect coculture with M2-polarized TAMs induced morphological changes in pancreatic cancer cells toward a fibroblast-like phenotype, in addition to increased expression of mesenchymal markers and decreased expression of epithelial markers. These results clearly demonstrated that coculture with M2-polarized TAMs dramatically promoted the process of EMT in pancreatic cancer cells regardless of their high or low potential of EMT.
Tumor cells often showed enhanced proliferation and mobility in addition to EMT. The two-chamber migration assays showed that M2-polarized TAMs promoted pancreatic cancer cell migration to the lower chamber. An MTT assay revealed increased proliferation of pancreatic cancer cells cocultured with M2-polarized TAMs over monocultured parental cells. Furthermore, proteolytic activities of MMP2 and MMP9 significantly increased in both Panc-1 and BxPC-3 cell lines, when cocultured with M2-polarized TAMs, and MMP9-mediated EMT was demonstrated. MMP9 is known to work with transcription factor snail to induce EMT in cervical carcinoma cells, ${ }^{23}$ which suggests that M2polarized TAMs increased the potential of EMT of pancreatic cancer cells.

What is the mechanism of M2-polarized TAM-mediated EMT in pancreatic cancer cells? TLR4 and specific ligands such as heat-shock proteins (HSP 60, 70), and nuclear protein high-mobility group box 1 (HMGB1) have previously been found within the tumor microenvironment. HMGB1 is significantly increased in pancreatic cancer, breast cancer and colon cancer, and this increase can promote activation of TLR4 on immune cells and induce cancer progression and metastasis. ${ }^{19}$ We hypothesized that TLR4 on M2-polarized TAMs promotes EMT, which leads to the aggressive behaviors of pancreatic cancer cells. To test this hypothesis, we induced knockdown or inhibition of TLR4 signaling in M2-polarized TAMs.

Knockdown of TLR4 by siRNA or treatment of TLR4neutralizing antibody significantly inhibited downregulation of E-cadherin and the increase of snail and vimentin. Furthermore, LPS-mediated activation of TLR4 signaling markedly increased EMT. Here, we demonstrated for the first time that TLR4 signaling on M2-polarized TAMs induced 
EMT in pancreatic cancer cells, introducing a novel cross-talk mechanism between stromal and tumor cells.

To confirm TLR4 signaling activity during EMT of pancreatic cancer cells, the role of the target gene of TLR4 signaling, IL-10, was investigated. Our results showed that the EMT of pancreatic cancer cells was markedly abolished by IL-10-neutralizing antibody, suggesting IL-10 is the mediator responsible for TLR4 signaling. In cancer patients with malignant melanoma, ovarian cancer, and other hematologic malignancies, such as lymphoma and multiple myeloma, IL-10 itself can be identified in the serum and/or tumor. Indeed, a negative correlation between circulating levels of IL-10 and prognosis has been reported. ${ }^{24}$ In the present study, we showed for the first time that IL-10 is the major mediator inducing EMT of pancreatic cancer cells, implying a potent target regarding to regulation of M2-polarized TAMs in tumor microenvironment.

To further clarify the role of TLR4/IL-10 signaling during the progression of pancreatic cancer, the proliferation, migration, and proteolytic activities of cancer cells were measured taking advantage of inhibition approaches of TLR4. As TLR4 siRNA and neutralizing antibodies of TLR4 and IL-10 significantly reversed the increased proliferation, migration, and proteolytic activities of cancer cells induced by M2-polarized TAMs, and LPS profoundly increased these neoplastic characteristics in coculture. Our findings suggest TLR4/IL-10 signaling participates in the development of pancreatic cancer.

Despite the interesting results of this study, there are certain limitations that should be highlighted and discussed. First, in the present, we only utilized the mouse macrophage RAW cell line in our experiments. To investigate conditions that would most represent those seen in humans, these experiments should be performed on primary cells, such as murine pancreatic cell lines or human monocytes. Second, although we identified specificity of M2-polarized TAMs through induction by IL-4 in this study, appropriate negative would include native or M1macrophages for all experiments performed. This is an especially important, given the relative novelty of the investigation of M2-polarized TAMs in EMT. Typical M1 macrophages produce high levels of IL-12 and IL-23, but exhibit low-level expression of IL-10. They also efficiently produce other cytokines, including IL- $1 \beta$, TNF- $\alpha$, and IL-6, and participate as inducers as well as effector cells in polarized Th1 responses, and mediate resistance against intracellular parasites and tumors. ${ }^{25}$ As such, for comparison and appropriate clarification of the roles of M2 macrophages in cellular transitions and other cell activities, native and M1macrophages should be included to rule out the influence of these macrophage types in such processes.

In conclusion, M2-polarized TAMs promoted EMT in pancreatic cancer cells, suggesting a novel mechanism by which M2-polarized TAMs contributed to the aggressive behavior of pancreatic cancer cells. Furthermore, TLR4/IL-10 signaling may contribute to the development of novel therapeutic strategies of pancreatic cancer.

\section{ACKNOWLEDGEMENTS}

This work was supported by grants from the Natural Science Foundation of Jiangsu Province (No.SBK201241438) and the key project of Nanjing Medical University (No.2010NJMUZ59).

\section{DISCLOSURE/CONFLICT OF INTEREST}

The authors declare no conflict of interest.

1. Hidalgo M. Pancreatic cancer. N Engl J Med 2010;362:1605-1617.

2. Gao Q, Zhao YJ, Wang XY, et al. CXCR6 upregulation contributes to a pro-inflammatory tumor microenvironment that drives metastasis and poor patient outcomes in hepatocellular carcinoma. Cancer Res 2012;72:3546-3556

3. Allavena P, Mantovani A. Immunology in the clinic review series; focus on cancer: tumor-associated macrophages: undisputed stars of the inflammatory tumor microenvironment. Clin Exp Immunol 2012;167:195-205.

4. Schmieder A, Michel J, Schönhaar K, et al. Differentiation and gene expression profile of tumor-associated macrophages. Semin Cancer Biol 2012:22:289-297.

5. Niino D, Komohara Y, Murayama T, et al. Ratio of M2 macrophage expression is closely associated with poor prognosis for angioimmunoblastic T-cell lymphoma (AITL). Pathol Int 2010;60:278-283.

6. Kurahara $H$, Shinchi $H$, Mataki $Y$, et al. Significance of $M 2$-polarized tumor-associated macrophage in pancreatic cancer. J Surg Res 2011;167:e211-e219.

7. Biddle A, Mackenzie IC. Cancer stem cells and EMT in carcinoma. Cancer Metastasis Rev 2012; (e-pub ahead of print).

8. Liu WF, Ji SR, Sun JJ, et al. CD146 expression correlates with epithelialmesenchymal transition markers and a poor prognosis in gastric cancer. Int J Mol Sci 2012;13:6399-6406.

9. Javle MM, Gibbs JF, Iwata KK, et al. Epithelial- mesenchymal transition (EMT) and activated extracellular signal-regulated kinase ( $p$-Erk) in surgically resected pancreatic cancer. Ann Surg Oncol 2007;14: 3527-3533.

10. Yadav A, Kumar B, Datta J, et al. IL-6 promotes head and neck tumor metastasis by inducing epithelial-mesenchymal transition via the JAKSTAT3-SNAIL signaling pathway. Mol Cancer Res 2011;9:1658-1667.

11. Condeelis J, Segall JE. Intravital imaging of cell movement in tumours. Nat Rev Cancer 2003;3:921-930.

12. Chen K, Huang J, Gong W, et al. Toll-like receptors in inflammation, infection and cancer. Int Immunopharmacol 2007;7:1271-1285.

13. Lee $\mathrm{CH}, \mathrm{Wu} \mathrm{CL}$, Shiau AL. Toll-like receptor 4 signaling promotes tumor growth. J Immunother 2010;33:73-82.

14. Banerjee $S$, Halder $K$, Bose $A$, et al. TLR signaling-mediated histone modification at IL-10 and IL-12 promoter region leads to functional impairments in tumor-associated macrophages. Carcinogenesis 2011;32:1789-1797.

15. Sato T, Terai M, Tamura Y. Interleukin 10 in the tumor microenvironment: a target for anticancer immunotherapy. Immunol Res 2011:51:170-182.

16. Lee $\mathrm{JH}$, Lee GT, Woo SH, et al. Bone morphogenetic protein-6 in renal cell carcinoma promotes tumor proliferation via M2 polarization of tumor-infiltrating macrophages through interleukin-10. Cancer Res; advance online publication, 30 April 2013 (e-pub ahead of print).

17. Monick MM, Robeff PK, Butler NS, et al. Phosphatidylinositol 3-kinase activity negatively regulates stability of cyclooxygenase 2 mRNA. J Biol Chem 2002;277:2992-3000.

18. Del Pozo JL. Primers on molecular pathways: lipopolysaccharide signaling-potential role in pancreatitis and pancreatic cancer. Pancreatology 2010;10:114-118.

19. Ellerman JE, Brown $C K$, de Vera $M$, et al. Masquerader: high mobility group box-1 and cancer. Clin Cancer Res 2007;13:2836-2848.

20. Zhou C, Nitschke AM, Xiong W, et al. Proteomic analysis of tumor necrosis factor-alpha resistant human breast cancer cells reveals a MEK5/Erk5-mediated epithelial-mesenchymal transition phenotype. Breast Cancer Res 2008;10:R105.

21. Biswas SK, Gangi L, Paul S, et al. A distinct and unique transcriptional program expressed by tumor-associated macrophages (defective NFkappaB and enhanced IRF-3/STAT1 activation). Blood 2006;107: 2112-2122. 
22. Dhar G, Mehta S, Banerjee $\mathrm{S}$, et al. Loss of WISP-2/CCN5 signaling in human pancreatic cancer: a potential mechanism for epithelial-mesenchymal transition. Cancer Lett 2007;254: 63-70.

23. Asuthkar S, Nalla AK, Gondi CS. Gadd45a sensitizes medulloblastoma cells to irradiation and suppresses MMP-9-mediated EMT. Neuro Oncol 2011;13:1059-1073.
24. Conti-Freitas LC, Foss-Freitas MC, Mamede RC, et al. Interferon-gamma and interleukin-10 production by mononuclear cells from patients with advanced head and neck cancer. Clinics 2012;67:587-590.

25. Tomioka H, Tatano Y, Maw WW, et al. Characteristics of suppressor macrophages induced by mycobacterial and protozoal infections in relation to alternatively activated M2 macrophages. Clin Dev Immunol 2012;2012:635451. 